Supporting Information

\title{
PtNPts/MWCNTs-PEDOT:PSS Modified
}

\author{
Microelectrode Arrays for the Synchronous
}

Dopamine and Neural Spike Detection in Rat

\section{Models of Sleep Deprivation}

Zeying Lu $u^{1,2}$, Shengwei Xu ${ }^{1,2}$, Hao Wang ${ }^{1,2}$, Enhui He ${ }^{1,2}$, Juntao Liu ${ }^{1,2}$, Yuchuan Dai ${ }^{1,2}$,

Jingyu Xie ${ }^{1,2}$, Yilin Song ${ }^{1,2}$, Yun Wang ${ }^{1,2}$, Yiding Wang ${ }^{1,2}$, Lina $Q u^{3, *}$, and Xinxia Cai ${ }^{1,2, *}$

${ }^{1}$ State Key Laboratory of Transducer Technology, Aerospace Information Research Institute,

Chinese Academy of Sciences, Beijing 100120, China;

${ }^{2}$ University of Chinese Academy of Sciences, Beijing 100042, China;

${ }^{3}$ State Key Laboratory of Space Medicine Fundamentals and Application, China Astronaut

Research and Training Center, Beijing 100094, China;

*Corresponding Emails: xxcai@mail.ie.ac.cn; linaqu@263.net. 
The fabricating and nanomaterial modification process of all the channels are the same, and all the sites own the competence to record the electrical signals and neural chemical signals. As shown in figure S1, the experimental devices we used to collect the neural signals have two modules, including electrical recording module and electrochemical recording module. In this study, the site 6 and 11 were connected to the electrochemical recording module of the device, and were designate as DA recording channels; While the other sites were connected to the electrical recording module of the device, and were designate as electrical recording channels to record spike and LFP. All of these channels can transmit the signal to their respective module so that the dual-mode neural detection can be realized.

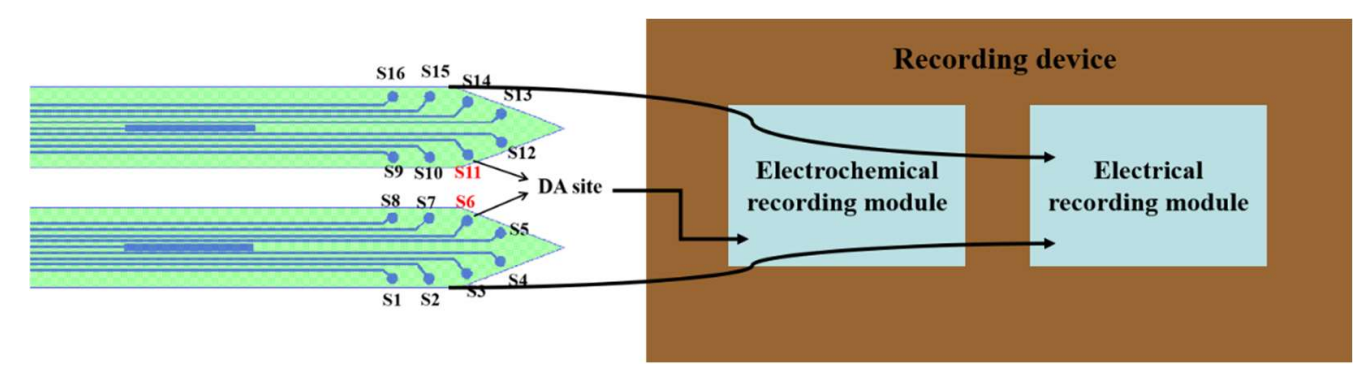

Figure S1. The different reocoding sites are connected to the different module of the recording device. The site 6 and 11 are connected to electrochemical recoding module, and the other sites are connected to electrical recording module.

The sensitivity is defined as the ratio between the output signal and measured property. And the sensitivity of the biosensor in this study is defined as:

$$
S=\Delta i / \Delta c
$$

where $\Delta \mathrm{i}$ represents the shift of the current the biosensor detected, and $\Delta \mathrm{c}$ stood for the concentration change of analyte. As shown in figure $6 \mathrm{a}$, from the in vitro calibration test, we plotted the relationship between the measured currents and concentrations of DA and fitted a straight line, mathematically represented as: 


\section{$Y=30.561 X+65.412$,}

where $\mathrm{X}$ represents the concentration of the DA, $\mathrm{Y}$ is the output current signal, and $30.561 \mathrm{pA} / \mu \mathrm{M}$ is the sensitivity of the biosensor.

As for the detection limit, it is referred to as the minimum amount of analyte that can be detected by a biosensor. In this study, the detection limit was defined by adding a low concentration of analyte where the signal-to-noise ratio (SNR) was not lower than 3 . The signal-to-noise $(\mathrm{S} / \mathrm{N})$ started to exceed 3 upon adding a 50 nM DA solution, which indicated that the detection limit was around $50 \mathrm{nM}$.

(a)

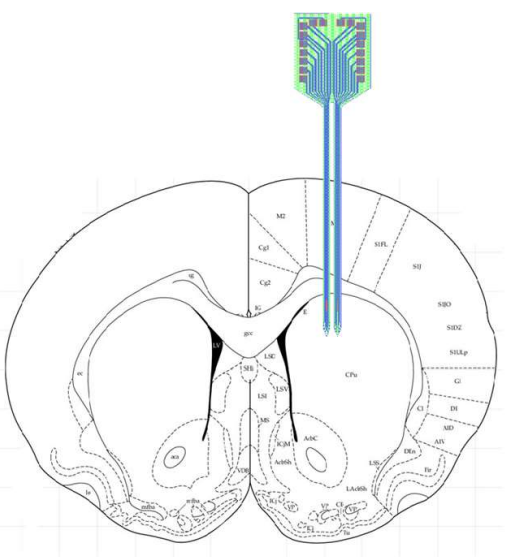

(c)

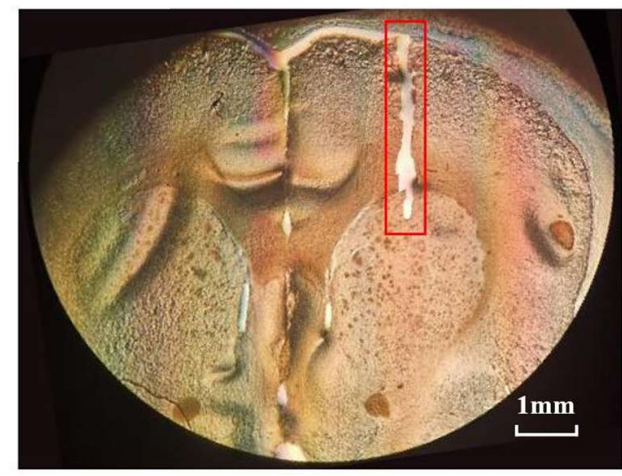

(b)

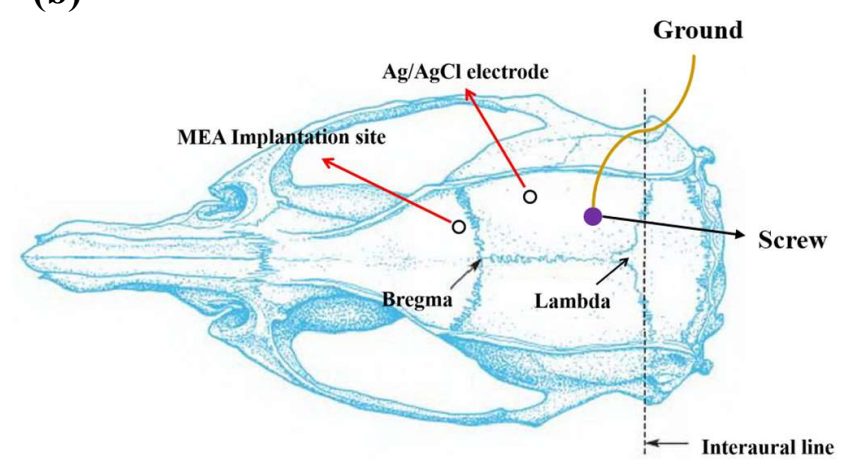

Figure S2. The implantation of MEA into rat brain.

(a) The rat brain structural map. The MEA was vertically implanted into the cortex and $\mathrm{CPu}$ of the right hemisphere using the micro-positioner. (b) The implantation position of the MEA located as AP, $0.96 \mathrm{~mm}$; ML, $2.4 \mathrm{~mm}$; DV, $-1.5 \mathrm{~mm}$ and $-3.8 \mathrm{~mm}$ ). $\mathrm{Ag} \mid \mathrm{AgCl}$ reference electrode is fixed beside the MEA. A screw on the scull was grounded via a copper wire. (c) The histological image with the trace of MEA implanted into the cortex and $\mathrm{CPu}$. 
(a)

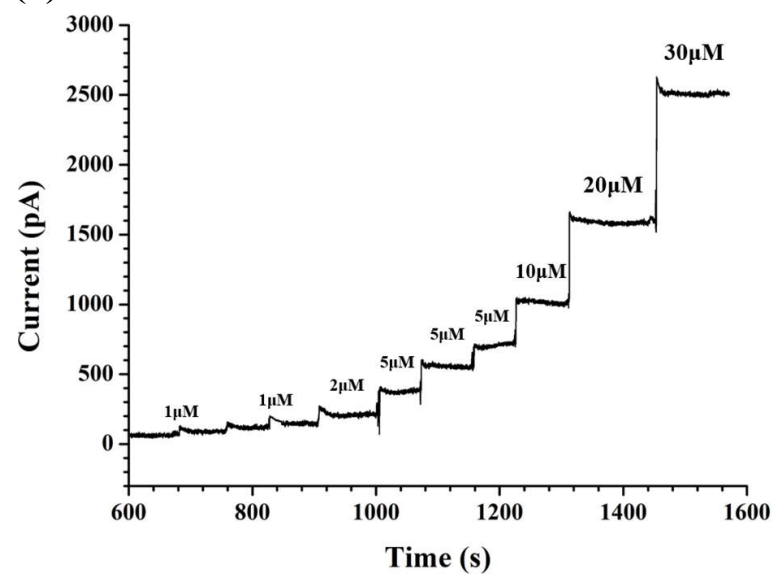

(b)

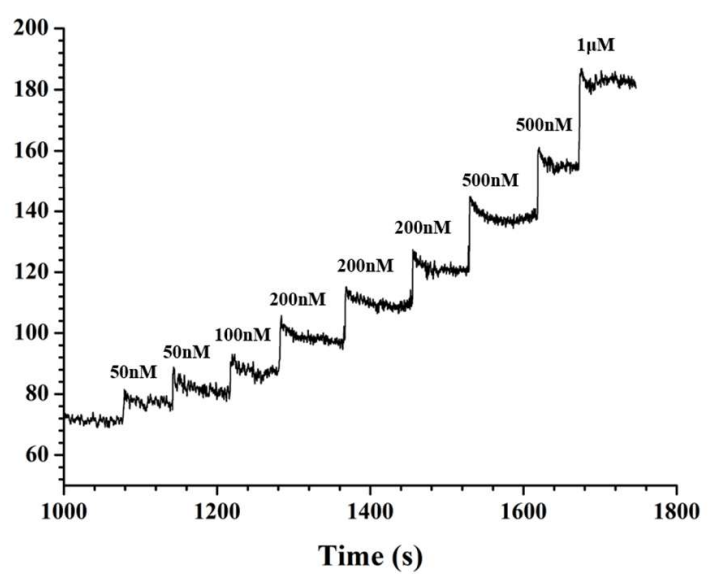

Figure S3. The calibration of DA electrodes by adding DA solution of different concentrations. (a) Calibration curve of one representative DA electrode measured at $0.2 \mathrm{~V}$ vs reference electrode by adding $1 \mu \mathrm{M}$ to $30 \mu \mathrm{M}$ DA solution. (b) Calibration curve of the electrode with the addition of low-concentration $(50 \mathrm{nM} \sim 1 \mu \mathrm{M}) \mathrm{DA}$.

Table S1: The repeatability of the PtNPts/MWCNts-PEDOT:PSS modified microelectrode.

\begin{tabular}{|c|c|c|c|c|c|}
\hline No. & 1 st & 2nd & 3rd & 4th & RSD (\%) \\
\hline $\begin{array}{c}\text { Sensitivity } \\
(\mathrm{pA} / \mu \mathrm{M})\end{array}$ & 30.362 & 29.871 & 30.796 & 31.238 & $1.92 \%$ \\
\hline
\end{tabular}

RSD: Relative Standard Deviation 


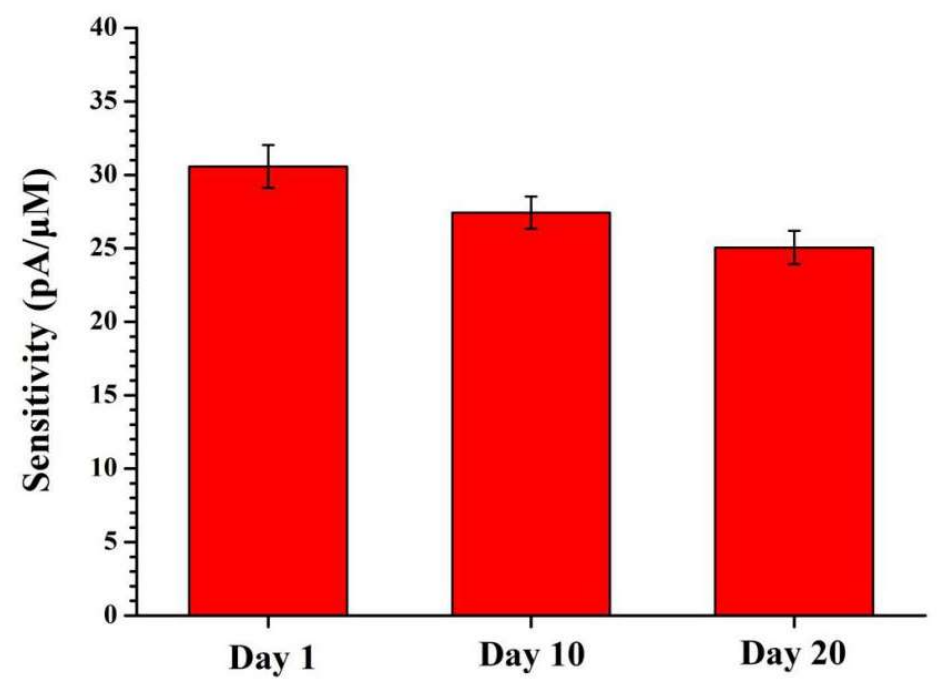

Figure S4. The sensitivity variation of PtNPts/MWCNts-PEDOT:PSS modified microelectrode in Day 1, Day 10 and Day 20 after the fabrication and modification. The sensitivity has decreased for $18 \%$ from $30.57 \mathrm{pA} / \mu \mathrm{M}$ to $25.06 \mathrm{pA} / \mu \mathrm{M}$ in twenty days. 